\title{
Encoded Hybrid DWT based Watermarking Scheme based on Singular Matrix Decomposition
}

\author{
Nikhil Nigam \\ Research Scholar \\ Computer Science and Engineering Department \\ Samrat Ashok Technological Institute Vidisha (M.P.)
}

\author{
Yogendra Kumar Jain \\ Head of Department \\ Computer Science and Engineering Department \\ Samrat Ashok Technological Institute Vidisha (M.P.)
}

\begin{abstract}
In the period of digital technology, digital information suffers from copyright and integrity violations. In case of any disputes like rights violation and content creator, digital watermark has a vital role to protect the property rights of the original owner of digital information. Numerous of watermarking techniques have been proposed recently but image quality of host image at receiving end is needed to be improved. We proposed Encoded Hybrid Digital Watermarking Scheme (EHDWS) to improve image quality which is based on Discrete Wavelet Transform (DWT), Singular Value Decomposition (SVD) and Bose ChaudhuriHocquenghem (BCH) code. DWT is used for decomposing the image into LL, HL, LH, HH sub-bands; SVD is used to decompose the image into orthogonal matrix (U), singular matrix $(\mathrm{S})$, and inverse orthogonal matrix $\left(\mathrm{V}^{\mathrm{T}}\right)$ and $\mathrm{BCH}$ code is used for error detection-correction, copyright protection and authentication. The proposed EHDWS has high degree of transparency, robustness, blindness. In EHDWS, watermark embedding scheme is used to encode the original watermark image by using $\mathrm{BCH}(7,4)$ based algorithm for obtaining the encoded watermark image. Then embed this encoded watermark image into host image by using DWT-SVD based algorithm for obtaining the watermarked image. Watermark extracting scheme is used to obtain both extracted watermark image for authentication purpose and extracted host image from watermarked image to obtain original image. PSNR values are also calculated to know the quality of watermarked image.
\end{abstract}

\section{General Terms}

Image security, watermarking.

\section{Keywords}

BCH Code, Digital Watermark, DWT, Haar Transform, SVD.

\section{INTRODUCTION}

In recent time, digital images can be captured effortlessly through scanners, digital camera and camcorders and transmitted easily in the Internet. As an outcome, digital images come into outlook widely in the internet and the World Wide Web (WWW) and in storage media for example CD-ROM and DVD. One of the most accepted image formats used is JPEG, which can get high compression while keeping high image quality. Related with the general transmission of images, it has the issues of copyright authentication and privacy. One possible result is to implant some unseen information into the images where the implanted information can be extracted for different purposes. Digital watermarking is a process to implant some information called watermark into dissimilar kinds of medium called Cover Work. Watermark is implanted by modifying the frequency coefficient of host image by using some common method like Discrete Fourier Transform (DFT), Discrete Cosine Transform (DCT), Discrete Wavelet Transform (DWT), etc. Watermark having numerous characteristics like Imperceptibility, transparency, secure, and robust in order to serve copyright protection, video authentication, and fingerprinting and copy control. There are two set of watermark for different application such as visible and invisible. There are much class of invisible watermarks for different applications such as fragile watermark and robust watermark. Fragile watermarks are intended to be broken easily by image processing operations. The broken watermark gives out as an indication of alteration of the original image. Major applications consist of tampering detection of images placed on the WWW and authentication of images obtained from suspicious sources. Robust watermarks are required to reside in the watermarked image even after it has been attacked. The attacks could be hostile attacks for example signal processing statistical averaging, watermark counterfeiting, watermark removal and estimation, etc. The attacks may probably be unintended attacks which are common image processing such as compression, cropping, filtering, scaling etc.

The DWT transformation scheme for the digital watermarking, which decomposes the input image into four components, that is, LL, HL, LH and $\mathrm{HH}$, where the first letter corresponds with frequency offset of the row either low or high and second letter refer to filter applied to the columns. The lowest resolution level LL mentions approximate part of the host image whereas rest three refer to detail parts and give the Vertical High (LH), Horizontal High (HL) and High (HH) frequencies. In the proposed algorithm, watermark is embedded into the host image by modifying the high frequency coefficients band i.e. HH sub band illustrated in figure 1. 


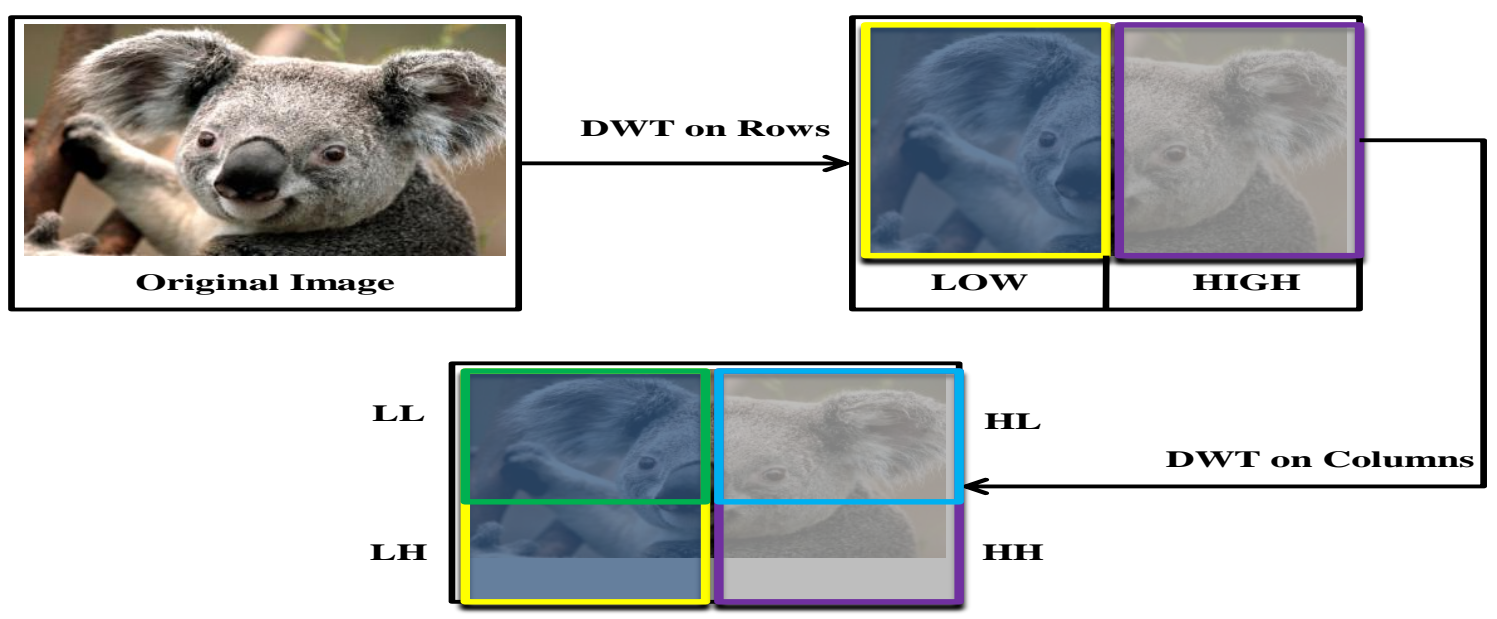

Fig 1: DWT transform of host image

SVD is based on a theorem from linear algebra which declares with the purpose of a rectangular matrix A can be broken into the product of three matrices - an orthogonal matrix (U), a singular matrix (S), and the inverse of an orthogonal matrix (V). The theorem is normally presented something like this: $\mathrm{A}=\mathrm{USV}^{\mathrm{T}}$, where $\mathrm{U}^{\mathrm{T}} \mathrm{U}=\mathrm{I}$ and $\mathrm{V}^{\mathrm{T}} \mathrm{V}=\mathrm{I}$; the columns of $\mathrm{U}$ are orthonormal eigenvectors of $\mathrm{AA}^{\mathrm{T}}$, the columns of $\mathrm{V}$ are orthonormal eigenvectors of $A^{T} A$ and $S$ is a singular matrix containing the square roots of Eigen matrix from $\mathrm{U}$ or $\mathrm{V}$ in descending order.

Generally BCH (Bose-Chaudhuri-Hocquenghem) code is used for error correction and detection, but in the proposed work, it is used for authentication and copyright protection purpose. $\mathrm{BCH}$ (Bose-Chaudhuri-Hocquenghem) code is a class of cyclic code which is accomplished for hit and miss error correction. The $\mathrm{BCH}$ technique used to transform each 4-bit data to a 7-bit data stream. For that reason, call $\mathrm{BCH}(7,4)$ code. Imagine with the objective of the authenticating data $\mathrm{a}$, is encoded to $\mathrm{c}$ by using BCH $(7,4)$ code. When c consists of a 1-bit error, we can decode it back to original data. When c consists of a 2-bit error, we cannot re-establish it but can observe the survival of the error. This procedure suggests a method with the purpose of, not only capable of authentication but also can re-establish altered authenticating data to its original form. A cyclic code has a good mathematical formation which holds the mechanism of automatic synchronization. This mechanism is given by a linear feedback shift register. If data is not correct for the period of decoding, it can rapidly come back to the state of regular decoding from the error state. This property is fairly robust for take-out data from a data stream that contains errors. We can get purpose of authenticating images with this advantage.

\section{RECENT WORK}

DWT is more appropriate for achieving robust and invisible watermarking scheme that leading to good visual quality watermarked image. For the past many years, the Singular Value Decomposition (SVD) is used as a new method for watermarking. It presents a common feature after changing an image and its structural information which plays a vital role in predicting the image quality. Amendments in singular vectors are associated to that in singular matrix which mainly represents the image luminance. We use DWT-SVD Hybrid Transform in the present work to carry out the watermark embedding.
$\mathrm{Fu}$ introduced the encoding method by using $\mathrm{BCH}$ code. In presented work, $\mathrm{BCH}$ code is used to encode the watermark image [1]. Al-Hashem et al. introduced the new binary (bit-level) lossless image compression method based on a well-known error correcting $\mathrm{BCH}$ code. The $\mathrm{BCH}$ encoder converts the message of $\mathrm{k}$ bits to a codeword of length $\mathrm{n}$ by eliminating 3 parity bits. In contrast the decoder adds these parity bits after verifying the received message [2]

Agarwal et al. introduced the optimized watermarking scheme based on the Discrete Wavelet Transform (DWT) and Singular Matrix Decomposition (SVD). In this scheme the singular matrix of a binary watermark are embedded in singular matrix of the LL3 sub-band coefficients of the host image by making use of Multiple Scaling Factors (MSFs) [3].

Munesh et al. introduced the DWT based watermarking. DWT is used to decompose the image into four sub-bands [4]. Liu et al. introduced the SVD based watermarking algorithm. In this algorithm, they compute singular matrix of the host image, transform it by embedding the watermark and apply SVD transform yet again on the key Matrix for finding the modified Singular Matrix. These singular matrix combine with watermark to extract the watermark image, the reverse process is applied for extracting watermark. Watermarking based on SVD already has been proposed by several researchers [7].

Li et al. introduced a hybrid DWT-SVD watermark scheme having human visual system. Hybrid DWT-SVD decomposes the host image into four sub-bands and applies SVD embedded singular matrix of the watermark into these sub-bands [8].

Chandra introduced a method for watermarking by adding singular matrix of the watermark to the singular matrix of the entire image. Initially, singular matrix of the host image and watermark are computed, and then magnified singular matrix of the watermark is added to host image [9].

Raval et al. introduce a multiple watermarking schemes based on DWT Transform. This decomposed the host image into two different row band LL, HH and embedded watermark over it. The scheme has a very high difference against the attacks like compression, noise addition, histogram equalization but not able to resist rotation, scaling and print-scan attacks [10]. 
Kasmani et al. introduced a hybrid transformation scheme which used both DWT and DCT to embed the digital watermark. Initially hybrid approach applied DCT to embed the watermark followed by three levels DWT decomposition. Hybrid scheme having good recovery against attacks but suffer from high time complexity problem [11].

Alarabeyyat et al. introduced the encoding method by using $\mathrm{BCH}$. In this scheme the well-known Lempel-Ziv-Welch (LZW) algorithm is used on the image. What comes out of the first step is forward to the second step where the Bose, Chaudhuri and Hoc-quenghem $(\mathrm{BCH})$ error correction and detected algorithm is used [12].

Loukhaoukha et al. introduced the optimal image watermarking algorithm based on LWT-SVD via multi-objective ant colony optimization [13]. Ishtiaq et al. introduced the adaptive watermark strength selection using particle swarm optimization. In their work authors used the optimization based watermarking scheme [14].

Xianghong et al. introduced the digital watermarking scheme based on DWT and vector transform for embed the watermark and extract the watermark for authentication with robustness [15].
In the presented work, an Encoded Hybrid Digital Watermarking scheme (EHDWS) has been proposed. EHDWS is based on DWT haar transformation, SVD and BCH $(7,4)$ code. DWT decompose both host image and watermark image into four frequency sub band namely LL, HL, LH and HH with help of haar transform. SVD decompose the HH sub-band of both host image and watermark image into orthogonal matrix $(\mathrm{U})$, singular matrix (S), and inverse orthogonal matrix $\left(\mathrm{V}^{\mathrm{T}}\right)$. $\mathrm{BCH}(7,4)$ encoder encodes the singular matrix $(\mathrm{S})$ of $\mathrm{HH}$ band of watermark image for authentication and copyright protection purpose.

Singular matrix (S) has value in diagonal, so we apply BCH (7, 4) code only on diagonal value which takes some time to perform action.

In this scheme we covert the double data type of singular image into integer data type and apply $\frac{p}{10}+64$ where $\mathrm{p}$ is pixel value of singular image. We do this so that it could come in range 64 to 127. Because we know that value in range 64-127 is represented in 7-bit. Our proposed work basically depends on 7-bit, because we apply the $\mathrm{BCH}(7,4)$ code on these 7 -bit to convert into 4-bit.

\subsection{Watermark Embedded Scheme}

\section{PROPOSED WATERMARKING SCHEME}

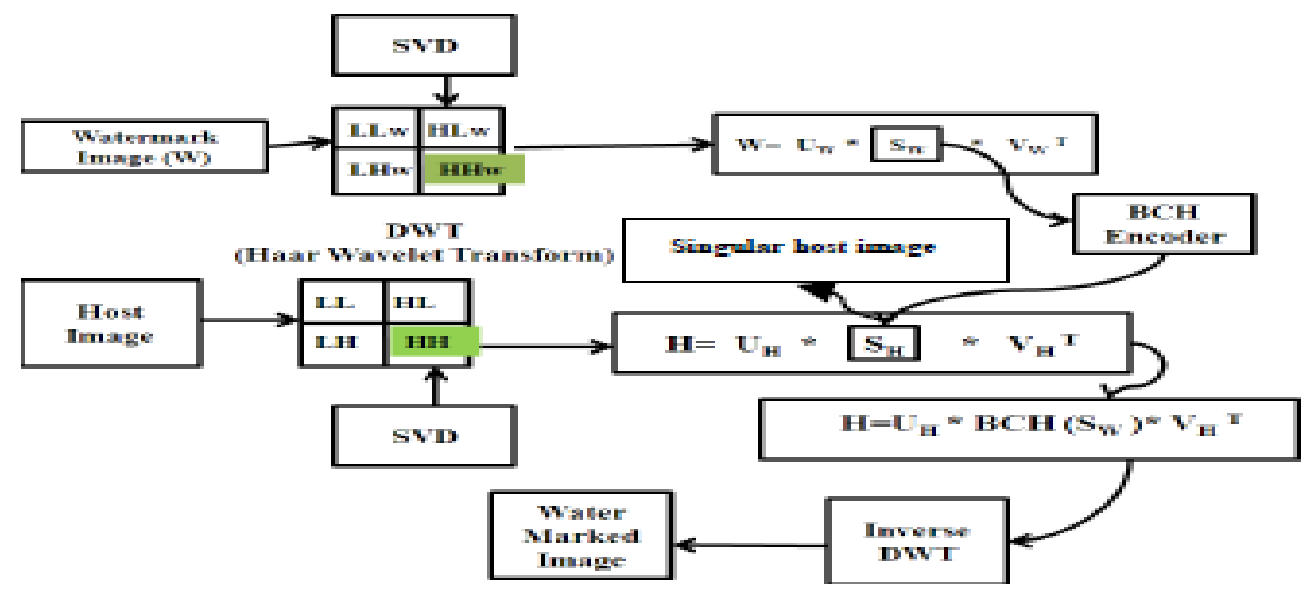

Fig 2: Proposed EHDWS watermark embedded scheme

As shown in figure 2, proposed watermark embedding scheme is based on both DWT and SVD with BCH $(7,4)$ encoder. Initially DWT decomposes both host image and watermark image into four frequency sub-bands namely LL, HL, LH, and HH band. LL band deals with approximate details, HL band deals with horizontal details, $\mathrm{LH}$ gives vertical details and $\mathrm{HH}$ band contain diagonal details of the images. Then apply SVD on $\mathrm{HH}$ band of both host image and watermark image to decompose the $\mathrm{HH}$ band into orthogonal matrix (U), singular matrix $(\mathrm{S})$ and Inverse orthogonal matrix $\left(\mathrm{V}^{\mathrm{T}}\right)$. We apply $\mathrm{BCH}$ $(7,4)$ encoder to encode the singular matrix $(\mathrm{S})$ of $\mathrm{HH}$ band of watermark image to obtain the encoded singular matrix (S'). In the proposed scheme, watermark is embedded by replacing the singular matrix (S) of $\mathrm{HH}$ band of host image with encoded singular matrix (S') of $\mathrm{HH}$ band of watermark image to obtain the watermarked image. Selection of watermark image in such a manner that it's singular Matrix (S) lies within the given range and energy of the singular matrix $(\mathrm{S})$ of watermark will be approximately equal to the energy of the singular matrix (S) of the host image. Hence the replacement of the singular matrix (S) will not affect perceptual quality of image. After this, we convert the singular matrix (S) of $\mathrm{HH}$ band of host image into singular host image, which has been used for extracting the host image at receiver end. In EHDWS, HH band has been used to embed the watermark, since it haves the fine details about image energy. In this way embedded watermark will not affect the perceptual fidelity of cover image.

\subsection{Watermark Extraction Scheme}




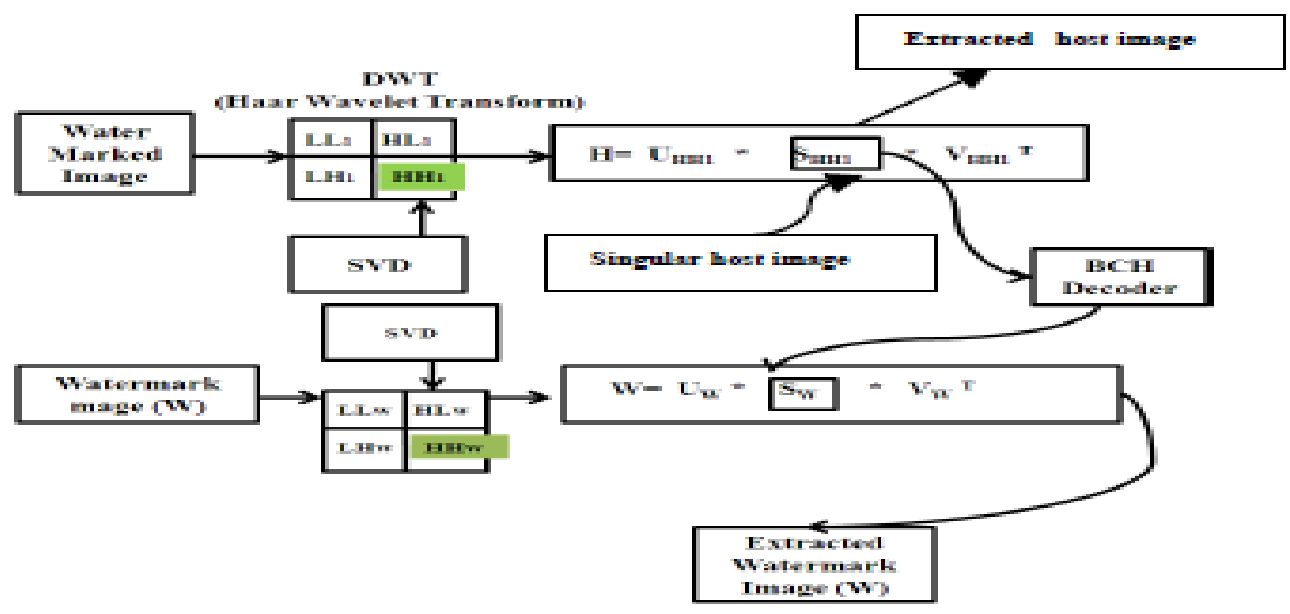

Fig 3: Proposed EHDWS watermark extraction scheme

As shown in figure 3, proposed watermark extracting scheme is based on both DWT and SVD with BCH $(7,4)$ decoder. Initially DWT decomposes both watermarked image and watermark image into four frequency sub-bands namely LL, HL, LH, and HH band. Then apply SVD on HH band of both watermarked image and watermark image to decompose the $\mathrm{HH}$ band into orthogonal matrix (U), singular matrix (S), and inverse orthogonal matrix $\left(\mathrm{V}^{\mathrm{T}}\right)$. We apply the $\mathrm{BCH}(7,4)$ decoder to decode the singular matrix (S) of $\mathrm{HH}$ band of watermarked image to obtain the decoded singular matrix (S). In The proposed scheme, watermark is extracted by replacing the singular matrix (S) of $\mathrm{HH}$ band of watermark image with decoded singular matrix (S) of $\mathrm{HH}$ band of watermarked image. If the watermark image and extracted watermark image is obtained similar to Original, it means authentication and copyright protection is successful. After this we covert singular host image which obtained at embedding scheme into singular matrix (S) of HH band of host image and put into the place of replaced decoded singular matrix (S') of $\mathrm{HH}$ band of watermarked image to obtain the extracted host image.

\subsection{Bose, Chaudhuri and Hocquenghem (BCH) Scheme}

3.3.1. $B C H(7,4)$ encoding scheme

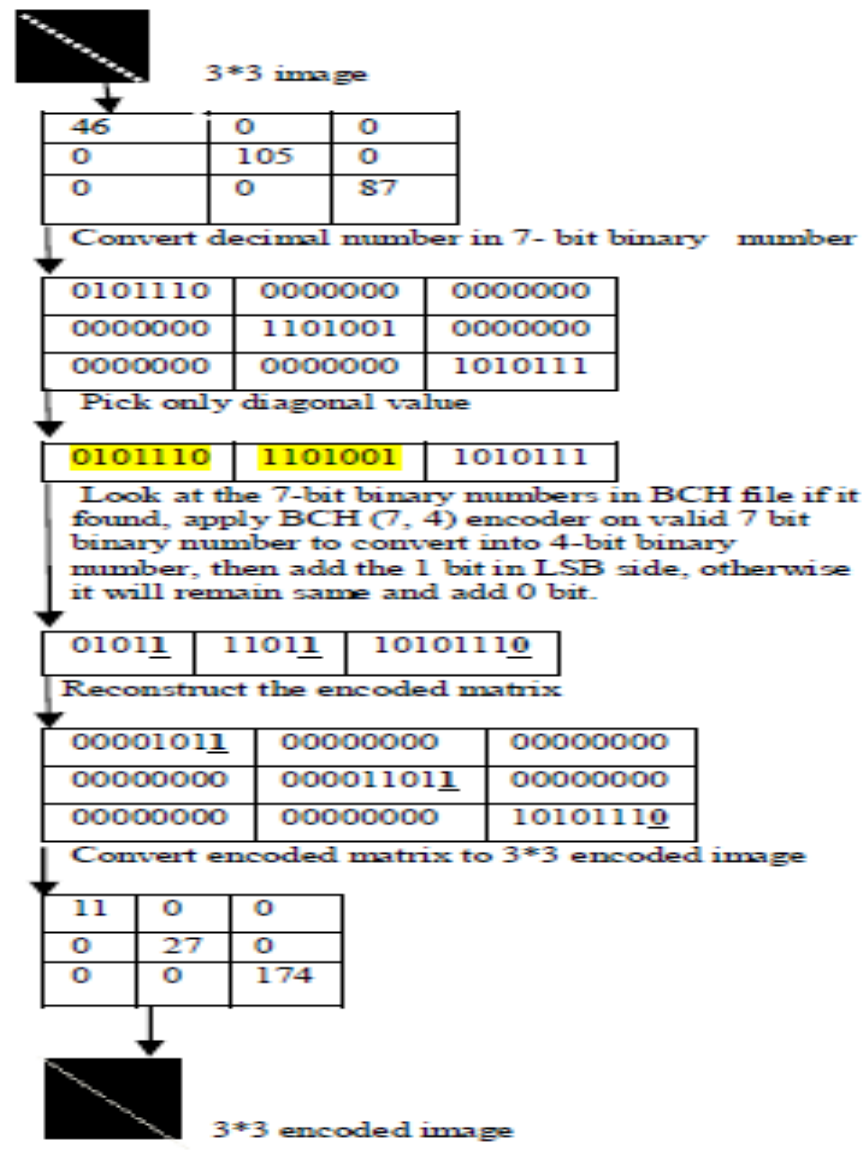

Fig 4: Proposed BCH $(7,4)$ encoding scheme 


\begin{tabular}{|ll|}
\hline 1. & 0000000 \\
\hline 2. & 0001001 \\
\hline 3. & 0010110 \\
\hline 4. & 0011101 \\
\hline 5. & 0100111 \\
\hline 6. & 0101100 \\
\hline 7. & 0110001 \\
\hline s. & 0111010 \\
\hline 9. & 1000101 \\
\hline 10. & 1001110 \\
\hline 11. & 1010011 \\
\hline 12. & 1011000 \\
\hline 13. & 1101001 \\
\hline 14. & 1110100 \\
\hline 15. & 1111111 \\
\hline
\end{tabular}

Fig 5: BCH file

$\mathrm{BCH}(7,4)$ encoding scheme presents the number of steps to encode the image. These Steps are as follows:

Step 1: Create a $\mathrm{BCH}$ file as shown in figure 5, which contain the list of 16 valid 7-bit binary numbers. Only these
16 valid 7- bit binary numbers will be encoded into 4-bit and other will remain same.

Step 2: Convert the original image into binary image which has 7-bit binary number's blocks.

Step 3: Pick only binary number's blocks of diagonal.

Step 4: Match binary number of each block with binary number of $\mathrm{BCH}$ file, if it match, we apply the $\operatorname{BCH}(7,4)$ encoder to convert each block of size 7 bits into a 4-bit length by removing 3 parity bit, then add the 1-bit in LSB side which is indicator for encoded blocks and if it not match, blocks will remain same bit size then adds only 0-bit which is indicator for un-encoded block.

Step 5: Reconstruct the encoded binary image.

Step 6: Convert this encoded binary image into encoded image.

\subsection{2. $\mathrm{BCH}(7,4)$ decoding scheme}

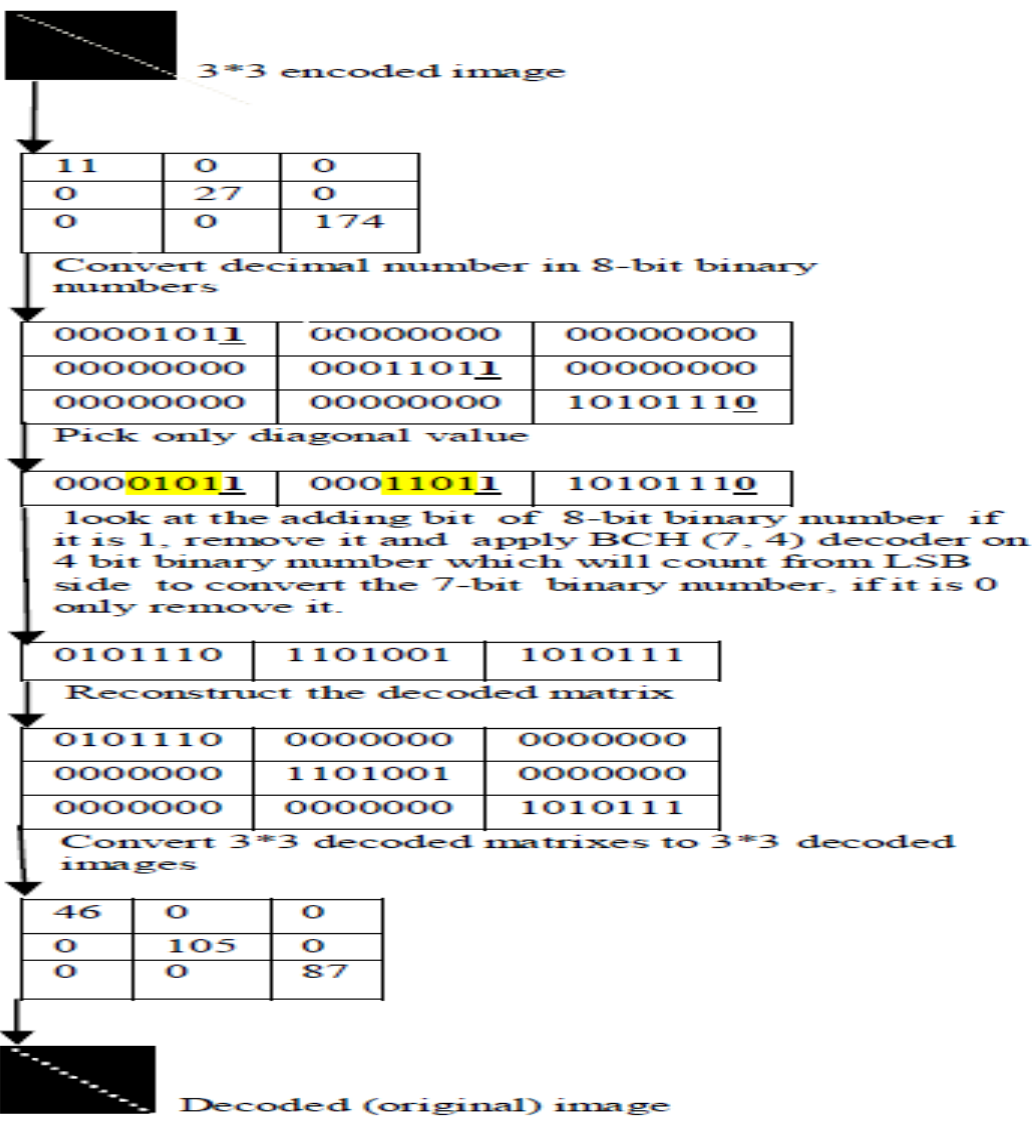

Fig 6: Proposed $\mathrm{BCH}(7,4)$ encoding scheme

In $\mathrm{BCH}(7,4)$ encoding scheme, we learnt about the encoding procedure which is used to construct the encoded image. Now we will learn about $\mathrm{BCH}(7,4)$ decoding procedure in $\mathrm{BCH}(7$, 4) decoding scheme, which is used to reconstruct the original image from the encoded image.

BCH $(7,4)$ decoding scheme also presents the number of steps to reconstruct the original image from the encoded image. These steps are as follows:
Step 1: Converts the encoded image into encoded binary image which has 8-bit binary number's blocks.

Step 2: Pick only binary number's blocks of diagonal.

Step 3: Look at the adding bit of 8-bit binary image in each block, if adding bit 1 , firstly remove it, then apply the $\mathrm{BCH}(7,4)$ decoders to convert 4-bit which is counted from LSB side into a 7-bit length by adding 3 parity bit and if adding bit 0 means block remain same bit size, remove only 0 bit. 
Step 4: Reconstruct the decoded binary image.

Step 5: Convert decoded binary image into original image.

Step 6: Finally, the image is returned to its original status without any loss of data.

\subsection{Algorithm}

\subsection{Watermark embedding algorithm}

1. $\left[\mathrm{LL}_{\mathrm{W}}, \mathrm{HL}_{\mathrm{W}}, \mathrm{LH}_{\mathrm{W}}, \mathrm{HH}_{\mathrm{W}}\right]=\mathrm{DWT}\left(\mathrm{WATERMARK} \mathrm{IMAGE}_{\mathrm{I}}\right)$

2. $\left[\mathrm{U}_{\mathrm{W}}, \mathrm{S}_{\mathrm{W}}, \mathrm{V}_{\mathrm{W}}^{\mathrm{T}}\right]=\operatorname{SVD}\left(\mathrm{HH}_{\mathrm{W}}\right)$

3. $[\mathrm{LL}, \mathrm{HL}, \mathrm{LH}, \mathrm{HH}]=\mathrm{DWT}\left(\mathrm{HOST}_{\mathrm{IMAGE}}\right)$

4. $\left[\mathrm{U}_{\mathrm{H}}, \mathrm{S}_{\mathrm{H}}, \mathrm{V}_{\mathrm{H}}^{\mathrm{T}}\right]=\mathrm{SVD}(\mathrm{HH})$

5. Singular host image $=\operatorname{IMWRITE}\left(\mathrm{S}_{\mathrm{H}}\right)$

6. $\mathrm{S}_{\mathrm{W}}{ }^{\mathrm{BCH}}=\mathrm{BCH} \_\mathrm{ENCODE}\left(\mathrm{S}_{\mathrm{W}}, 4\right)$

7. $\mathrm{HH}_{\mathrm{WM}}=\operatorname{ISVD}\left(\mathrm{U}_{\mathrm{H}} * \mathrm{~S}_{\mathrm{W}}{ }^{\mathrm{BCH}} * \mathrm{~V}_{\mathrm{H}}{ }^{\mathrm{T}}\right)$

8. WATERMARKED ${ }_{\text {IMAGE }}=\operatorname{IDWT}\left(\mathrm{LL} * \mathrm{HL} * \mathrm{LH}^{*} \mathrm{HH}_{\mathrm{WM}}\right.$

\subsection{Watermark extracting algorithm}

1. $\left[\mathrm{LL}_{\mathrm{W}}, \mathrm{HL}_{\mathrm{W}}, \mathrm{LH}_{\mathrm{W}}, \mathrm{HH}_{\mathrm{W}}\right]=\mathrm{DWT}\left(\mathrm{W} A T E R M A R K_{\mathrm{IMAGE}}\right)$

2. $\left[\mathrm{U}_{\mathrm{W}}, \mathrm{S}_{\mathrm{W}}, \mathrm{V}_{\mathrm{W}}^{\mathrm{T}}\right]=\operatorname{SVD}\left(\mathrm{HH}_{\mathrm{W}}\right)$
3. $\left[\mathrm{LL}_{1}, \mathrm{HL}_{1}, \mathrm{LH}_{1}, \mathrm{HH}_{1}\right]=\mathrm{DWT}\left(\mathrm{WATERMARKED} \mathrm{IMAGE}_{\text {I }}\right)$

4. $\left[\mathrm{U}_{\mathrm{HH} 1}, \mathrm{~S}_{\mathrm{HH} 1}, \mathrm{~V}_{\mathrm{HH} 1}^{\mathrm{T}}\right]=\operatorname{SVD}\left(\mathrm{HH}_{1}\right)$

5. $\mathrm{S}_{\mathrm{W} 1}=$ BCH_DECODE $\left(\mathrm{S}_{\mathrm{HH} 1}, 4\right)$

6. $\mathrm{HH}_{\mathrm{W} 1}=\operatorname{ISVD}\left(\mathrm{U}_{\mathrm{W}} * \mathrm{~S}_{\mathrm{W} 1}{ }^{\prime} * \mathrm{~V}_{\mathrm{W}}{ }^{\mathrm{T}}\right)$

7. EXTRACTED WATERMARK $\mathrm{IMAGE}_{\mathrm{I}}$ $=\operatorname{IDWT}\left(\mathrm{LL}_{\mathrm{W}}, \mathrm{HL}_{\mathrm{W}}, \mathrm{LH}_{\mathrm{W}}, \mathrm{HH}_{\mathrm{W} 1}\right)$

8. We Compare WATERMARK IMAGE $_{\text {and EXTRACTED }}$ WATERMARK $_{\text {IMAGE }}$, if both images same means authentication and copyright protection is successful.

9. $\quad \mathrm{S}_{\mathrm{HH}}=\operatorname{IMREAD}($ Singular host image $)$

10. $\mathrm{HH}_{0}=\operatorname{ISVD}\left(\mathrm{U}_{\mathrm{HH} 1}, \mathrm{~S}_{\mathrm{HH}}, \mathrm{V}_{\mathrm{HH} 1}{ }^{\mathrm{T}}\right)$

11. EXTRACTED HOST $\left.\operatorname{IMAGE}_{\mathrm{IDWT}}=\mathrm{ILL}_{1}, \mathrm{HL}_{1}, \mathrm{LH}_{1}, \mathrm{HH}_{\mathrm{O}}\right)$

\section{RESULT ANALYSIS}

The proposed works has been tested on the different images of size 256x256. Lena, Pepper and Baboon images has been used and all images are in color. The selected watermark images and host images are of the same size. Simulation work is carried out on the MATLAB tool on the i3 processor with 4 GB RAM and 500 GB HDD.

Table1: Comparison between PSNR ration of various approach

\begin{tabular}{|c|c|c|}
\hline image name & Algorithm & PSNR (dB) \\
\hline \multirow[t]{6}{*}{ Lena } & Proposed Approach & 56.8189 \\
\hline & $\begin{array}{l}\text { Single Scaling } \\
\text { Factor }\end{array}$ & 53.3062 \\
\hline & $\begin{array}{l}\text { Multiple Scaling } \\
\text { Factors }\end{array}$ & 55.7296 \\
\hline & $\begin{array}{l}\text { Loukhaoukha et al. } \\
\text { (2011) }\end{array}$ & 47.718 \\
\hline & Ishtiaq et al (2010) & 48.105 \\
\hline & $\begin{array}{l}\text { Xianghong et al } \\
(2004)\end{array}$ & 49.075 \\
\hline \multirow[t]{6}{*}{ Peppers } & Proposed Approach & 58.1941 \\
\hline & $\begin{array}{l}\text { Single Scaling } \\
\text { Factor (2014) }\end{array}$ & 52.09 \\
\hline & $\begin{array}{l}\text { Multiple Scaling } \\
\text { Factors (2014) }\end{array}$ & 52.15925 \\
\hline & $\begin{array}{l}\text { Loukhaoukha et al. } \\
\text { (2011) }\end{array}$ & 48.097 \\
\hline & Ishtiaq et al (2010) & NA \\
\hline & $\begin{array}{l}\text { Xianghong et al. } \\
(2004)\end{array}$ & 49.075 \\
\hline \multirow[t]{6}{*}{ Baboon } & Proposed Approach & 67.6462 \\
\hline & $\begin{array}{l}\text { Single Scaling } \\
\text { Factor (2014) }\end{array}$ & 53.0487 \\
\hline & $\begin{array}{l}\text { Multiple Scaling } \\
\text { Factors (2014) }\end{array}$ & 50.76746 \\
\hline & $\begin{array}{l}\text { Loukhaoukha et al. } \\
\text { (2011) }\end{array}$ & 52.379 \\
\hline & Ishtiaq et al (2010) & 44.9624 \\
\hline & $\begin{array}{l}\text { Xianghong et al. } \\
(2004)\end{array}$ & 49.075 \\
\hline
\end{tabular}

The expression Peak Signal-to-Noise Ratio, often abbreviated PSNR, which represent ratio of maximum possible power of a signal and the power of corrupting noise that affects the fidelity. PSNR can be describing in term of logarithmic scale. Higher
PSNR would indicate lower fidelity and vice versa. This would occur because we wish to minimize the MSE (Mean Square Error) between images with respect the maximum signal value of the image. The mean squared error for our practical purposes allows us to compare the "true" pixel values of our original 
image to our degraded image. A comparison of our proposed work with the different researcher in the recent past has been shown in table 1 , it can be easily said that the proposed algorithm works best with the DWT-SVD-BCH algorithm.

PSNR and MSE are calculated by the following formulas.

$$
\begin{aligned}
P S N R & =10 \log _{10}\left(\frac{M A X^{2}}{M S E}\right) \ldots \ldots \\
M S E & =\frac{\sum_{M, N}\left[I_{1}(m, n)-I_{2}(m, n)\right]^{2}}{M * N}
\end{aligned}
$$

The experimental results presented in table 1 depicted that PSNR range is between $48 \mathrm{db}-53 \mathrm{db}$ for all the existing technique between host image and watermarked image. DWTSVD based Firefly Algorithm (FA) technique having approximate $53.3062 \mathrm{db}$ PSNR in single scale and $55.7296 \mathrm{db}$ PSNR in multi scale for Lena, $52.09 \mathrm{db}$ PSNR in single scale and $52.15925 \mathrm{db}$ PSNR in multi scale for Peppers and 53.0487 $\mathrm{db}$ PSNR in single scale and $50.76746 \mathrm{db}$ PSNR in multi scale for Baboon which is greatest among all the existing approach $i$. e. Loukhaoukha et al. having approximate $47.718 \mathrm{db}$ PSNR for Lena, approximate $48.097 \mathrm{db}$ PSNR for Peppers and $52.379 \mathrm{db}$ PSNR for Baboon by using LWT-SVD via multi-objective ant colony optimization based algorithm. Ishtiaq et al. having approximate $48.105 \mathrm{db}$ PSNR for Lena and $44.9624 \mathrm{db}$ PSNR for Baboon by using particle swarm optimization based algorithm. Xianghong et al. having approximate $49.075 \mathrm{db}$ PSNR for Lena, 49.075db PSNR for Peppers and $49.075 \mathrm{db}$ PSNR for Baboon by using DWT and vector transform based algorithm whereas proposed DWT-SVD based BCH $(7,4)$ code authentication watermarking scheme having approximate $56.8189 \mathrm{db}$ PSNR for Lena, 58.1941 db PSNR for Peppers and $67.6462 \mathrm{db}$ PSNR for Baboon. Hence proposed algorithm provides the better performance with comparison to previous approaches.

The experimental results shows that the proposed algorithm gives the better performance with compared to previous approaches. The results are also better for Lena, Peppers and baboon images which were also used by previous researchers.
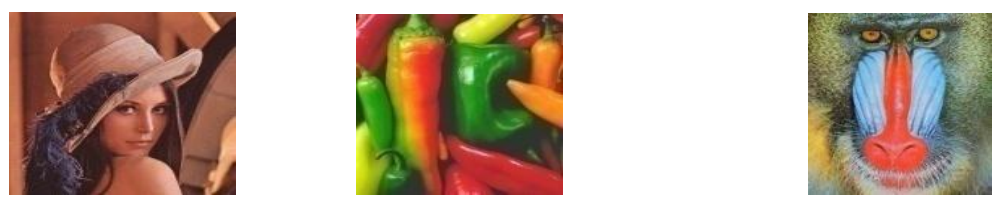

Fig 7: Host images

Fig. 7 shows the three host image on which the watermark image been use. In this figure Lena, Peppers and Baboon images are will be embedded. In this work, there are different images has host image.

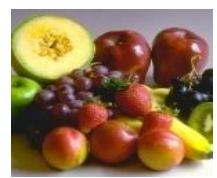

Fig 8: Watermark image

Fig. 8 shows the watermark image which will embed on host used Fruit image as a watermark image. Both images will take as images after applying $\mathrm{BCH}(7,4)$ encoder. In this figure only we an input using the MATLAB code.
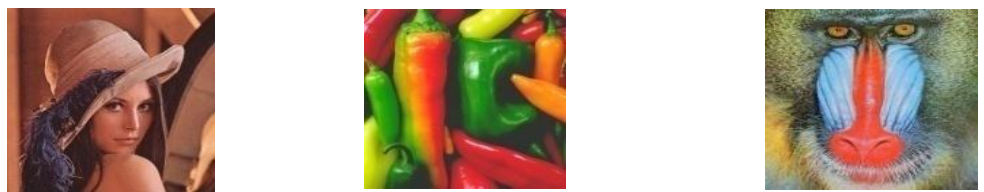

Fig 9: Watermarked images

Fig. 9 shows watermarked image which is transferred from one end to another end.

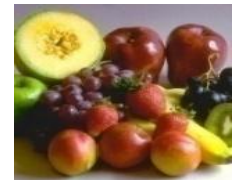

Fig 10: Extracted watermark image

Fig. 10 shows extracted watermark image from watermarked image after applying $\mathrm{BCH}(7,4)$ decoder which is used authentication purpose.
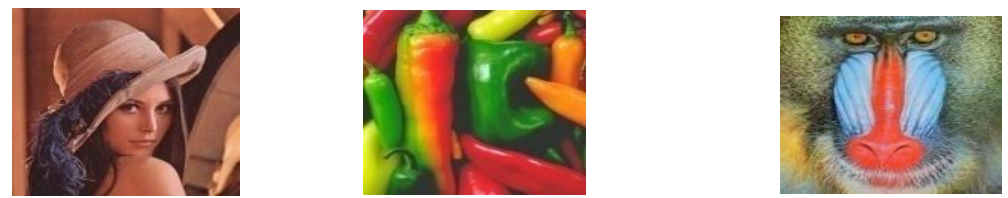

Fig 11: Extracted host images 
Fig. 11 shows extracted host image from watermarked image which is the original host image

\section{CONCLUSION AND FUTURE WORK}

In this dissertation digital watermarking Scheme (EHDWS) has been proposed. Proposed framework for watermarking scheme which is based on DWT and SVD transforms with BCH code based authentication. EHDWS is based on DWT HAAR transformation with higher band Singular value decomposition. In presented EHDWS firstly we DWT decompose both host image and watermark image into four frequency sub band namely LL, HL, LH and HH with help of HARR transform. Then SVD calculate singular value of $\mathrm{HH}$ band of host and watermark image, and then apply $\mathrm{BCH}$ code of singular value of watermark image into host image to send to receiving side. Receiving side use the inverse process to extract the watermark. This scheme contains property of blindness, transparency, robustness. In the proposed Encoded Hybrid Digital Watermarking Scheme (EHDWS), BCH code and DWT-SVD transformations provide both high degree of robustness and high degree of transparency because it doesn't affect visual quality of host image. In presented work, visual quality is shown by PNSR value, while watermark extracting scheme provide high degree of blindness because it does not use the host image to extract the watermark image.

In this scheme, we applied $\frac{p}{10}+64$ equation where $\mathrm{p}$ is pixel value of singular image. We do this so that it could come in range 64-127 for representing the value in 7-bit. Our scheme only applicable for max value 630 of singular matrix, because above 630, binary number will be increase from 7-bit to 8-bit, then $\mathrm{BCH}(7,4)$ will not be applicable on it. For that reason, in future time we will develop smart equation to convert for all range of pixel value in 7 bit.

In future time complexity of proposed work to embed and extract watermark is try to be minimized and try to apply some cryptography technique to maintained security of watermark.

In EHDWS, BCH $(7,4)$ code has been used for authentication and copyright protection not for error detection and correction. In future, $\mathrm{BCH}(7,4)$ code may be used for error detection and correction to obtain the original image from noisy image. Here EHDWS has been applied only in image, not in video. In future it may be used in video.

\section{ACKNOWLEDGMENTS}

I would like to thank Dr. Yogendra Kumar Jain, Head, Department of Computer Science and Engineering, who has contributed towards development of the template.

\section{REFERENCE}

[1] Yonggang $\mathrm{Fu}, 2013$ "Robust oblivious image watermarking scheme based on coefficient relation", Elsevier on Optic, Vol. 124, pp. 517-521.

[2] Rafeeq Al-Hashemi, Israa Wahbi Kamal, 2011 “A New Lossless image Compression Technique Based on Bose, Chandhuri and Hocquengham", International Journal of Software Engineering and Its Applications, Vol. 5, pp xxx.
[3] Charu Agarwal, Anurag Mishra, Arpita Sharma, 2014 "Optimized gray-scale image watermarking using DWTSVD and Firefly Algorithm", Elsevier on Expert Systems with Applications, Vol. xx, pp. xxx.

[4] Chandra Munesh, Pandey Shikha, 2010 "A DWT Domain Visible watermarking Techniques for Digital images", International Conference on Electronics and Information Engineering, Vol. 2, pp. 421-427.

[5] Sathik M. Mohammed and Sujatha S. S, 2010 "An Improved Invisible watermarking Technique for image Authentication", International Journal of Advanceed Science and Technology, Vol. 24, pp. 61-74.

[6] Hazem A. Al-Otum, Allam O. Al-Taba'a, 2009 “Adaptive color image watermarking based on a modified improved pixel-wise masking technique", IEEE transaction of Computers and Electrical Engineering, Vol. 35, pp. 673 695.

[7] Liu, F., \& Liu, Y., 2008 “A watermarking algorithm for digital image based on DCT and SVD", IEEE Congress on image and signal processing, Vol. 1, pp. 380-383.

[8] Li, Q., Yuan, C., Zong, Y. Z. , 2007 “Adaptive DWTSVD domain image watermarking using human visual model", ICACT, pp. 1947-1951.

[9] Chandra, D. S., 2002 "Digital image watermarking using singular matrix decomposition", Proceedings of the $45^{\text {th }}$ Midwest symposium on circuits and systems (MWSCAS'02), Vol. 3, pp. 264-267.

[10] Raval M. S. and Rege P. P. , 2003 "Discrete Wavelet Transform based multiple watermarking scheme", TENCON, Conference on Convergent Technologies for Asia-Pacific Region, Vol. 3, No. 1, pp. 935-938.

[11] Kasmani S A and Naghsh-Nilchi A , 2008 "A new robust digital image watermarking technique based on joint DWT-DCT Transformation", $3^{\text {rd }}$ International Conference on Convergence and Hybrid Information Technology ICCIT '08, Vol. 2, No. 1, pp. 539-544.

[12] Alarabeyyat, Al-Hashemi1, T. Khdour1, 2012 "Lossless image Compression Technique Using Combination Methods", Journal of Software Engineering and Applications, Vol. 5, pp. 752-763.

[13] Loukhaoukha, K., Chouinard, J.-Y., \& Taieb, M. H., 2011 "Optimal image watermarking algorithm based on LWTSVD via multi-objective ant colony optimization”, Journal of Information Hiding and Multimedia Signal Processing, Vol. 2, No. 4, pp. 303-319.

[14] Ishtiaq, M., Sikandar, B., Jaffar, A., \& Khan, A., 2010 "Adaptive watermark strength selection using particle swarm optimization", ICIC Express Letters, Vol. 4, No. 5,

[15] Xianghong, T., Lu, L., Lianjie, Y., \& Yamei, N., 2004 “A digital watermarking scheme based on DWT and vector transform" Proceeding of international symposium on intelligent multimedia, video and speech processing, pp. $635-638$ 